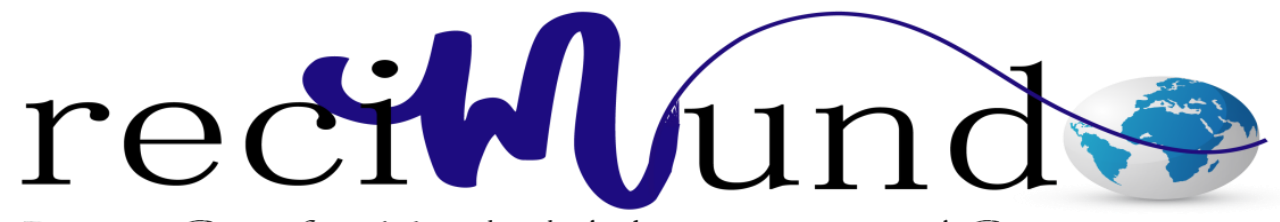

Revista Científica Mundo de la Investigación y el Conocimiento

Jorge Fernando Mena Terán ${ }^{\text {a }}$; Christian Eduardo Almendáriz Rodríguez ${ }^{\text {b; Felipe }}$ Gonzalo Naranjo Calderón ${ }^{\text {c; }}$ Nelly Virginia Mena Guerrero d

Medición y control del porcentaje de desperdicios de los materiales de construcción de la estructura de un galpón o nave industrial, ubicado en la vía Duran-Tambo

Revista Científica Mundo de la Investigación y el Conocimiento. Vol. 2 núm.2, mayo, ISSN: 2588-073X, 2018, pp. 183-193

DOI: $10.26820 /$ recimundo/2.(2).2018.183-193

Editorial Saberes del Conocimiento

Recibido: 05/12/2017

Aceptado: $15 / 02 / 2018$

a. puchimisterio@ @ hotmail.com

b. Universidad de Guayaquil; christian.almendarizr@ug.edu.ec

c. fgnaranjo@gmail.com

d. nellymen310@hotmail.com 


\section{Medición y control del porcentaje de desperdicios de los materiales de construcción de la estructura de un galpón o nave industrial, ubicado en la vía Duran-Tambo}

Vol. 2, núm. 2., (2018)

Jorge Fernando Mena Terán; Christian Eduardo Almendáriz Rodríguez; Felipe Gonzalo Naranjo Calderón; Nelly Virginia Mena Guerrero

\section{RESUMEN}

Es importante resaltar hoy en día que la Ingeniería Civil marca el desarrollo de las grandes ciudades en el mundo convirtiéndose en una de las mayores fuentes de trabajo en nuestro país y el mundo en general. El sector de la construcción ha priorizado las construcciones de rápida ejecución, uno de los rubros más controlados por el personal técnico es el de la mano de obra; en cualquier tipo de construcción, se pierde material al ejecutar cada actividad. El ejecutar un proyecto u obra, se generan pérdidas de materiales, debido a que es una fabricación manual, por eso no se puede controlar con precisión el consumo de material porque cada proceso constructivo posee un tipo diferente de desperdicio. Esta indagación está fundamentada en los objetivos esenciales que es la reducción del costo de adquisición de los materiales y la reducción de los desperdicios originados en la construcción del proyecto, por consiguiente, se aplica la teoría de Lean Construccion. Este análisis nos va a coadyuvar en la medición y control de los materiales estimados preeminentes por su importe en el mercado y sus usos dependiendo de las actividades a ejecutarse como: Zapatas Corridas, Zapatas Aisladas, Columnas, Muro Portante (encofrado, preparación del acero, hormigonado in situ), Estructura de Cubierta (cerchas), en la construcción de la estructura de un galpón o nave industrial; para lo cual se realizara una supervisión en dichos insumos y se establece los indicadores por originar los sobrantes acorde se va ejecutando y estableciendo los procedimiento de mejora en el proyecto; obteniendo como resultado la reducción del factor real del porcentaje (\%) de los materiales especificados en esta proposición logrando suscitar una rentabilidad para la empresa constructora delegada para el desarrollo del proyecto.

Palabras clave: Fabricación, constructivo, técnico, obra, proyecto, rubros. 


\section{Medición y control del porcentaje de desperdicios de los materiales de construcción de la estructura de un galpón o nave industrial, ubicado en la vía Duran-Tambo}

Vol. 2, núm. 2., (2018) Jorge Fernando Mena Terán; Christian Eduardo Almendáriz Rodríguez; Felipe Gonzalo Naranjo Calderón; Nelly Virginia Mena Guerrero

\section{ABSTRACT}

It is important to highlight today that Civil Engineering marks the development of large cities in the world becoming one of the largest sources of work in our country and the world in general. The construction sector has prioritized the construction of rapid execution, one of the items most controlled by the technical staff is that of the workforce; In any type of construction, material is lost when executing each activity. The execution of a project or work, material losses are generated, because it is a manual manufacture, so you can not precisely control the consumption of material because each construction process has a different type of waste. This investigation is based on the essential objectives that is the reduction of the cost of acquisition of the materials and the reduction of the waste originated in the construction of the project, therefore, the theory of Lean Construccion is applied. This analysis will help us in the measurement and control of the preeminent estimated materials by their amount in the market and their uses depending on the activities to be executed as: Running shoes, insulated shoes, columns, supporting walls (formwork, preparation of steel, concreting in situ), Roof structure (trusses), in the construction of the structure of a warehouse or industrial warehouse; for which a supervision will be carried out in said inputs and the indicators are established by originating the surpluses that are being executed and establishing the improvement procedures in the project; obtaining as a result the reduction of the real factor of the percentage (\%) of the materials specified in this proposal, achieving a profitability for the construction company delegated for the development of the project.

Key words: Manufacturing, constructive, technical, work, project, items. 


\section{Medición y control del porcentaje de desperdicios de los materiales de construcción de la estructura de un galpón o nave industrial, ubicado en la vía Duran-Tambo}

Vol. 2, núm. 2., (2018)

Jorge Fernando Mena Terán; Christian Eduardo Almendáriz Rodríguez; Felipe Gonzalo Naranjo Calderón; Nelly Virginia Mena Guerrero

\section{Introducción.}

Es importante mencionar que el sector de la construcción, es quien más utiliza los recursos naturales como son: la madera, minerales, energía y agua. Teniendo en cuenta que los cálculos ingenieriles nos demuestran datos sobre los materiales a usarse en una y otra actividad en nuestros presupuestos y análisis de costos de las obras de construcción civil.

Se considera que estos datos deben ser afectados por un factor de desperdicio, sin embargo, los ingenieros tratan de controlar los materiales o insumos, es decir, tratar de reducir el porcentaje de los desperdicios generados en obra siendo muy creativos en el desarrollo de los procesos constructivos.

Los materiales de construcción representan un $25 \%$ al $30 \%$ del costo de un proyecto. Mediante este estudio nos vamos orientando a la metodología para el consumo de materiales para las partidas de control teniendo en cuenta los insumos de mayor costo que representan en la ejecución del proyecto estos pueden ser: el acero estructural, cemento, encofrado, etc. Debemos tener en cuenta q no debe de haber excedentes en los materiales solicitados.

La presente investigación tiene como objetivo Plasmar una guía para el control de desperdicios de materiales de construcción en un galpón; mediante cálculos comparativos y el cálculo de materiales conjeturados en los planos del proyecto, para poder optimizar los recursos, calidad de estos y el valor final del proyecto en la construcción de un galpón o nave industrial ubicado en el km 6,5 km de la vía Duran-Tambo. 
Medición y control del porcentaje de desperdicios de los materiales de construcción de la estructura de un galpón o nave industrial, ubicado en la vía

Duran-Tambo

Vol. 2, núm. 2., (2018)

Jorge Fernando Mena Terán; Christian Eduardo Almendáriz Rodríguez; Felipe Gonzalo Naranjo

Calderón; Nelly Virginia Mena Guerrero

\section{Metodología.}

Técnicas para la obtención de información

Entrevista

Es un coloquio en donde se plantean las interrogantes bajo un manual desarrollado con anterioridad, en un orden sensato y se declaran de la misma forma como han sido formuladas. Todo el procedimiento del dialogo esta preliminarmente dispuesto y no hay albedrio para romper lo programado. Las preguntas que se realizaron a los Ingenieros y Arquitectos son de forma cerrada; lo que significa que las respuestas son limitadas.

Según menciona (Frances, 2017)

Una entrevista es básicamente una conversación entre dos personas, pero en investigación cuantitativa esta entrevista tiene una peculiaridad fundamental: a todos los sujetos que se entrevista se les pregunta lo mismo y de la misma forma, y las respuestas que se obtienen de las preguntas son registradas también de la misma forma.

Reglas para una entrevista estructurada

Al realizar la entrevista estructurada debemos ubicarnos en una posición cómoda desde el punto de vista físico y psicológico del entrevistado. 


\section{Medición y control del porcentaje de desperdicios de los materiales de construcción de la estructura de un galpón o nave industrial, ubicado en la vía Duran-Tambo}

Vol. 2, núm. 2., (2018)

Jorge Fernando Mena Terán; Christian Eduardo Almendáriz Rodríguez; Felipe Gonzalo Naranjo Calderón; Nelly Virginia Mena Guerrero

Las interrogantes deben realizarse tal cual están en el formulario previamente realizado y con una ecuanimidad posible.

Transcribir las resoluciones los más precisos y vertiginosamente posible para que la persona entrevistada conserve la calma.

No se puede sugerir ningún tipo de respuestas ni comentarios sobre las preguntas formuladas.

Ventaja de una entrevista estructurada

- $\quad$ Obtener un testimonio más específico e íntegro.

- $\quad$ Información o datos reales.

- $\quad$ Faculta obtener datos de personas de cualquier nivel cultural.

- $\quad$ Oportunidad de disipar interrogantes

Desventajas de la entrevista estructurada

- $\quad$ Requieren de una mayor cantidad de tiempo.

- El personal encargado en realizar la entrevista debe ser preparado y conocer todo lo relacionado al tema a preguntar.

\section{Cuestionario.}

El cuestionario se denomina también formulario o guía de entrevista; este documento comprende una sucesión de interpelaciones que son realizadas por el personal encargado de la 


\section{Medición y control del porcentaje de desperdicios de los materiales de construcción de la estructura de un galpón o nave industrial, ubicado en la vía Duran-Tambo}

Vol. 2, núm. 2., (2018) Jorge Fernando Mena Terán; Christian Eduardo Almendáriz Rodríguez; Felipe Gonzalo Naranjo Calderón; Nelly Virginia Mena Guerrero

entrevista al entrevistador; lo primordial en este sistema es saber qué clase de cuestionario a utilizar. El tipo de formulario se lo entregara personalmente ya que la entrevista es dirigida.

Partes del cuestionario: debe comprender el nombre de la entidad o institución que auspician el proyecto; la intención de la exploración, el aprovechamiento que obtendría la persona encuestada y la sociedad; para finalizar el pedido afable de cooperación.

1 ¿Considera usted que la falta de planificación, control y Experiencia por parte del personal técnico y la cuadrilla de obrero; son los principales causantes del mal uso de los materiales de construcción?

2 ¿Se podría decir que el sobredimensionamiento de la cuadrilla del personal de trabajo es una de las causas para que el índice de desperdicio incremente?

3 L ¿Cree usted que las empresas están preparadas para solucionar el mal aprovechamiento de los materiales asignados para determinado proyecto?

4 ¿Usted está de acuerdo que la calidad de los materiales influye en el índice de desperdicio?

5 Cree usted que la inadecuada ubicación de las instalaciones temporales del proyecto como: baños, garitas, almacenes o bodegas, etc. ¿Sea una de las causales en el incremento de los desperdicios de los materiales de construcción? 
Medición y control del porcentaje de desperdicios de los materiales de construcción de la estructura de un galpón o nave industrial, ubicado en la vía Duran-Tambo

Vol. 2, núm. 2., (2018)

Jorge Fernando Mena Terán; Christian Eduardo Almendáriz Rodríguez; Felipe Gonzalo Naranjo Calderón; Nelly Virginia Mena Guerrero

\section{Resultados.}

Los entrevistados fueron Ingenieros y Arquitectos; quienes tienen una ardua experiencia.

En la tabla $\mathrm{N}^{\circ} 1$ se aprecian las preguntas realizadas a los entrevistados antes mencionados.

\section{Tabla $N^{\circ}$ 1.- Preguntas Realizadas}

\begin{tabular}{c|c|l|} 
VALOR & \multicolumn{1}{c}{ NÚMEROS \% } \\
\hline SI & 10 & $100 \%$ \\
NO & 0 & $0 \%$ \\
TOTAL & 10 & $100 \%$ \\
\hline
\end{tabular}

Al desarrollar esta interpelación en el desarrollo de la entrevista, todos los entrevistados están de acuerdo que la falta de experiencia del personal técnico y la cuadrilla de trabajadores provoca el aumento del desperdicio por el inadecuado uso de los materiales.

Tabla $N^{\circ}$ 2.- Exceso de Personal

\begin{tabular}{c|c|c} 
VALOR & NÚMEROS & $\%$ \\
\hline SI & 7 & $70 \%$ \\
\hline NO & 3 & $30 \%$ \\
\hline TOTAL & 10 & $100 \%$ \\
\hline
\end{tabular}


Medición y control del porcentaje de desperdicios de los materiales de construcción de la estructura de un galpón o nave industrial, ubicado en la vía

Duran-Tambo

Vol. 2, núm. 2., (2018)

Jorge Fernando Mena Terán; Christian Eduardo Almendáriz Rodríguez; Felipe Gonzalo Naranjo

Calderón; Nelly Virginia Mena Guerrero

El $70 \%$ de los profesionales entrevistados están de acuerdo que el exceso de personal afecta al auge del desperdicio de los componentes del proyecto, el otro $30 \%$ de los interrogados manifiestan que hay actividades en las cuales se requieren más obreros que en otras.

\section{Tabla $N^{\circ}$ 3.- Organización}

\begin{tabular}{c|c|c|}
\multicolumn{2}{c}{ VALOR } & \multicolumn{2}{c}{ NÚMEROS } & $\%$ \\
\hline SI & 10 & $100 \%$ \\
\cline { 2 - 3 } NO & 0 & $0 \%$ \\
\cline { 2 - 3 } TOTAL & 10 & $100 \%$
\end{tabular}

El $100 \%$ de las personas relacionadas con la industria de la construcción, están totalmente de acuerdo, que la empresa designada está en la facultad de asumir estos gastos, ya que antes de entregar un presupuesto final al dueño del proyecto, se realizan los respectivos análisis de los precios unitarios APU.

\section{Tabla $\mathbf{N}^{\circ}$ 4.- Capacitación del Personal}

\begin{tabular}{c|c|c|}
\multicolumn{2}{c}{ VALOR } & \multicolumn{2}{c}{ NÚMEROS } & $\%$ \\
\hline SI & 6 & $60 \%$ \\
NO & 4 & $40 \%$ \\
\cline { 2 - 3 } TOTAL & 10 & $100 \%$ \\
\cline { 2 - 3 } & &
\end{tabular}

Al cuestionar sobre la calidad de los materiales de construcción el 60 \% de los Ingenieros Civiles cuestionados están conforme que esta cualidad es una de las causas del agravamiento de 


\section{Medición y control del porcentaje de desperdicios de los materiales de construcción de la estructura de un galpón o nave industrial, ubicado en la vía Duran-Tambo}

Vol. 2, núm. 2., (2018)

Jorge Fernando Mena Terán; Christian Eduardo Almendáriz Rodríguez; Felipe Gonzalo Naranjo Calderón; Nelly Virginia Mena Guerrero

los desperdicios, el $40 \%$ manifiesta que depende del material ya que se lo pueden reutilizar según la actividad a ejecutarse en el proyecto.

Tabla $N^{\circ}$ 5.- Ubicación de Materiales

\begin{tabular}{c|c|c} 
VALOR & NÚMEROS & $\%$ \\
\hline SI & 10 & $100 \%$ \\
\hline NO & 0 & $0 \%$ \\
\hline TOTAL & 6 & $100 \%$ \\
\hline
\end{tabular}

El $100 \%$ de los profesionales citados afirma, que la mala ubicación de dichas instalaciones, no solo trae como consecuencia el incremento del índice de desperdicio de los materiales, sino que también afecta al tiempo de productividad del proyecto.

\section{Conclusiones.}

Se debe gozar de una buena comunicación entre los residentes de la obra que pueden ser Ingenieros o Arquitectos, los encargados de la parte administrativas y los obreros; para asegurar la clase de los procesos constructivos y el manejo de los materiales.

El tipo de manufactura, es decir, la mano de obra prevalece en el origen de los desperdicios de los materiales; ya que son los obreros los encargados en realizar los elementos para el encofrado y las piezas del acero de refuerzo que se necesitan en la ejecución del proyecto. 


\section{Medición y control del porcentaje de desperdicios de los materiales de construcción de la estructura de un galpón o nave industrial, ubicado en la vía \\ Duran-Tambo}

Vol. 2, núm. 2., (2018)

Jorge Fernando Mena Terán; Christian Eduardo Almendáriz Rodríguez; Felipe Gonzalo Naranjo

Calderón; Nelly Virginia Mena Guerrero

La insuficiente supervisión o fiscalización; por parte de los Ingenieros o Arquitectos residentes de la obra por parte los contratistas, pueden hacer tomar una determinación errada a los obreros en el desarrollo de alguna actividad.

El diseño y construcción de los proyectos en Ingeniería Civil son muy engorrosos; por eso es sustancial contar con un sistema de disminución de desperdicios en los materiales de construcción.

\section{Bibliografía.}

Alarcón, L. (1997). Herramientas para identificar y reducir perdidas en proyectos de construcción. Ingenieria de Construccion, 15(1), 37-45.

Duda, W. (1977). Cemento Manual Tecnologico. Barcelona: Técnicos Asociados.

Novas Cabrera, J. (2010). Sistemas constructivos prefabricados aplicables a la construccion de edificaciones. Madrid: Universidad Politécnica de Madrid.

Salazar, S. (2005). Costo y tiempo en edificación. México: Limusa Noriega.

Universidad EIA. (2017). Estructuras compuestas por elementos tipo cercha. Antioquia: Escuela de Ingeniería de Antioquia. 